\title{
Recruitment of focal adhesion kinase and paxillin to $\beta$ I integrin promotes cancer cell migration via mitogen activated protein kinase activation
}

\author{
David L Crowe* and Arthur Ohannessian
}

Address: Center for Craniofacial Molecular Biology, University of Southern California, 2250 Alcazar Street, Los Angeles, CA 90033, USA

Email: David L Crowe* - dcrowe@usc.edu; Arthur Ohannessian - aohannes@ucla.edu

* Corresponding author

Published: 07 May 2004

BMC Cancer 2004, 4:18

This article is available from: http://www.biomedcentral.com/I47/-2407/4/18

(C) 2004 Crowe and Ohannessian; licensee BioMed Central Ltd. This is an Open Access article: verbatim copying and redistribution of this article are permitted in all media for any purpose, provided this notice is preserved along with the article's original URL.

\begin{abstract}
Background: Integrin-extracellular matrix interactions activate signaling cascades such as mitogen activated protein kinases (MAPK). Integrin binding to extracellular matrix increases tyrosine phosphorylation of focal adhesion kinase (FAK). Inhibition of FAK activity by expression of its carboxyl terminus decreases cell motility, and cells from FAK deficient mice also show reduced migration. Paxillin is a focal adhesion protein which is also phosphorylated on tyrosine. FAK recruitment of paxillin to the cell membrane correlates with Shc phosphorylation and activation of MAPK. Decreased FAK expression inhibits papilloma formation in a mouse skin carcinogenesis model. We previously demonstrated that MAPK activation was required for growth factor induced in vitro migration and invasion by human squamous cell carcinoma (SCC) lines.
\end{abstract}

Methods: Adapter protein recruitment to integrin subunits was examined by coimmunoprecipitation in SCC cells attached to type IV collagen or plastic. Stable clones overexpressing FAK or paxillin were created using the lipofection technique. Modified Boyden chambers were used for invasion assays.

Results: In the present study, we showed that FAK and paxillin but not Shc are recruited to the $\beta I$ integrin cytoplasmic domain following attachment of SCC cells to type IV collagen. Overexpression of either FAK or paxillin stimulated cancer cell migration on type IV collagen and invasion through reconstituted basement membrane which was dependent on MAPK activity.

Conclusions: We concluded that recruitment of focal adhesion kinase and paxillin to $\beta I$ integrin promoted cancer cell migration via the mitogen activated protein kinase pathway.

\section{Background}

Integrin extracellular domains bind extracellular matrix molecules such as type IV collagen and laminin while the cytoplasmic domains bind specific components of the actin cytoskeleton [1]. Integrin-extracellular matrix interactions also activate signaling cascades such as mitogen activated protein kinases [2]. Integrin binding to extracel- lular matrix or integrin crosslinking increases tyrosine phosphorylation of focal adhesion kinase (FAK) [3]. FAK consists of a central catalytic domain and amino and carboxyl terminal non- catalytic domains. A focal adhesion targeting sequence within the carboxyl terminus is required for localization to focal adhesions [4]. FAK undergoes autophosphorylation on a single tyrosine 
residue which creates a binding site for $\mathrm{SH} 2$ containing proteins. Inhibition of FAK activity by expression of its carboxyl terminus decreases cell motility, and cells from FAK deficient mice also show reduced migration $[5,6]$.

Paxillin is a focal adhesion protein which is also phosphorylated by a number of stimuli on tyrosine [2]. Paxillin interacts with FAK at two sites in the amino and carboxyl termini which are not required for targeting paxillin to focal adhesions [7]. A mutant paxillin protein lacking both FAK binding sites exhibits reduced tyrosine phosphorylation [8]. FAK recruitment of paxillin to the cell membrane correlates with Shc phosphorylation and activation of the MAPKs ERK and JNK [9]. However JNK phosphorylation is not dependent on FAK nor on phosphatidylinositol 3-kinase (PI3K) activity.

Transformation of FAK deficient fibroblasts with the v-Src oncogene promotes cellular motility equal to that of FAK re-expression [10]. However, these cells are not invasive and required intact FAK expression to produce this phenotype. Decreased FAK expression inhibits papilloma formation in a mouse skin carcinogenesis model [11]. These studies indicate that FAK has a key role in tumorigenesis and invasion. We previously demonstrated that PI3K, ERK, and JNK activation were required for growth factor induced in vitro migration and invasion by human squamous cell carcinoma (SCC) lines $[12,13]$. These studies led us to hypothesize that FAK and paxillin may mediate signals from the substratum via ERK in order to regulate cell migration. In the present study, we show that FAK and paxillin but not Shc are recruited to the $\beta 1$ integrin cytoplasmic domain following attachment of SCC cells to type IV collagen. This recruitment correlated with increased cellular migration on this substratum. Overexpression of either FAK or paxillin stimulated cancer cell migration on type IV collagen and migration through reconstituted basement membrane which was dependent on ERK activity. We concluded that recruitment of focal adhesion kinase and paxillin to $\beta 1$ integrin promoted cancer cell migration and invasion via the mitogen activated protein kinase pathway.

\section{Methods \\ Cell culture and stable transfection}

SCC4 and SCC25 cells were cultured in Dulbecco's modified Eagle medium (DMEM), 10\% fetal bovine serum, 40 $\mu \mathrm{g} / \mathrm{ml}$ gentamicin at $37^{\circ} \mathrm{C}$ in a humidified atmosphere of $5 \% \mathrm{CO}_{2}$. Cells were plated on tissue culture plastic dishes or commercially available plates coated with type IV collagen (Becton Dickinson). Some cells were pretreated with blocking antibodies to $\beta 1$ or $\beta 4$ integrins (Invitrogen) for 1 hour prior to plating $[14,15]$. Other cultures were deprived of attachment by plating on uncoated petri dishes in media containing $1.6 \%$ methylcellulose. Cells were transfected with $5 \mu \mathrm{g}$ expression vector for FAK or paxillin (kindly provided by Dr. Thomas Parsons) or neomycin resistance plasmid alone using Lipofectamine reagent according to manufacturer's recommendations (Invitrogen). Cells were selected in $400 \mu \mathrm{g} / \mathrm{ml} \mathrm{G} 418$ for 14 days. Resistant clones were picked for expansion and characterization. FAK and paxillin expression was determined by western blot.

\section{Immunoprecipitation}

Cultures were lysed in $50 \mathrm{mM}$ HEPES (pH 7.5), $150 \mathrm{mM}$ $\mathrm{NaCl}, 1 \mathrm{mM}$ EDTA, $2.5 \mathrm{mM}$ EGTA, $1 \mathrm{mM}$ DTT, 1\% Nonidet P-40, 10\% glycerol, $1 \mathrm{mM} \mathrm{NaF}, 0.1 \mathrm{mM}$ sodium orthovanadate, and protease inhibitors for 30 minutes at $4^{\circ} \mathrm{C}$. Lysates were centrifuged at $10,000 \times \mathrm{g}$ for 10 minutes and anti- $\beta 1$ integrin antibody (Invitrogen) was incubated with the supernatants for 1 hour at $4^{\circ} \mathrm{C}$. Antigen-antibody complexes were precipitated with protein $\mathrm{A} / \mathrm{G}$ agarose beads for 1 hour at $4^{\circ} \mathrm{C}$. Samples were boiled in Laemmli buffer for 3 minutes, separated by SDS-PAGE, and blotted to polyvinylidene difluoride membranes. Blots were incubated with anti-FAK, paxillin (Transduction Laboratories), Shc, and Grb2 (Santa Cruz Biotechnology) antibodies to determine relative association with $\beta 1$ integrin. Blots were then incubated with anti- $\beta 1$ integrin antibody to ensure equal amounts of immunoprecipitated protein in each lane. Bands were analyzed by laser densitometry and the numerical data subjected to $t$ test.

\section{Western blot}

$75 \mu \mathrm{g}$ total cellular protein was separated by SDS-PAGE on $10 \%$ resolving gels under denaturing and reducing conditions. Separated proteins were electroblotted to PVDF membranes according to manufacturer's recommendations (Roche Molecular Biochemicals). Blots were incubated with antibodies to human FAK, paxillin, ERK1, activated ERK1 (Transduction Laboratories), or $\beta 1$ integrin (Invitrogen) for 16 hours at $4{ }^{\circ} \mathrm{C}$. After washing in Tris buffered saline containing $0.1 \%$ Tween 20 (TBST, pH 7.4), blots were incubated for 30 minutes at room temperature with anti-IgG secondary antibody conjugated to horseradish peroxidase. Following extensive washing in TBST, bands were visualized by the enhanced chemiluminescence method (Roche Molecular Biochemicals). Bands were analyzed by laser densitometry and the numerical data subjected to $t$ test.

\section{Migration and invasion assays}

Cells were plated at confluence on plastic in the presence or absence of type IV collagen for 24-48 hours. Some cells were pretreated with blocking antibodies to $\beta 1$ or $\beta 4$ integrins for 1 hour prior to plating. Other cultures were treated with $50 \mu \mathrm{M}$ PD98059, a selective inhibitor of ERK activation. The dishes were scored with a pipet tip across the center of the plate. The number of cells which 
A

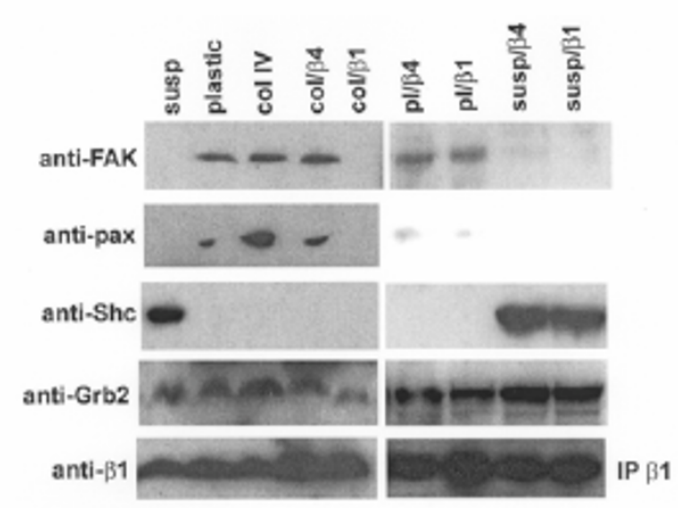

B

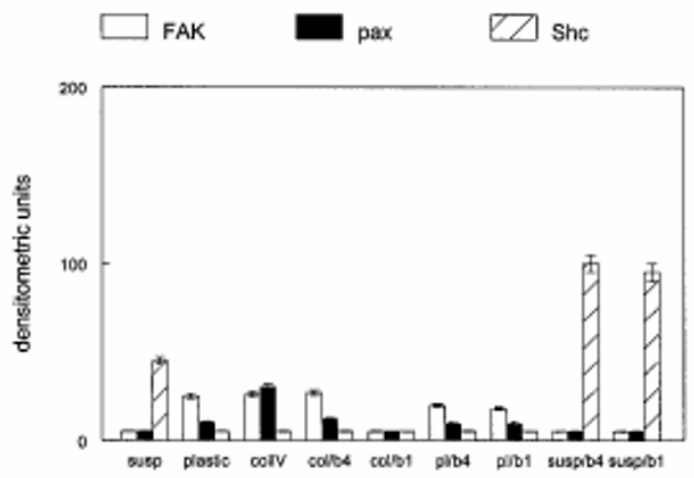

Figure I

FAK and paxillin immunoprecipitate with $\beta I$ integrin following cell attachment to type IV collagen. SCC25 cells were plated in semisolid media (susp), on tissue culture plastic ( $\mathrm{pl}$ ), or on type IV collagen (col) for 2 hours. Some cultures were preincubated with antibodies which block attachment by $\beta$ I or $\beta 4$ integrin subunits. $\beta$ I integrin was immunoprecipitated (IP $\beta$ I) from cell lysates and western blotted using the antibodies indicated at left. These experiments were performed three times with similar results. Representative blots are shown. (B) Densitometric quantitation of blots in Fig. IA.

migrated into the blank area of the dish during the specified time period was determined by counting of 10 random fields using phase contrast microscopy. For invasion assays, $2 \times 10^{5}$ cells were plated in triplicate into commercially available Matrigel invasion chambers (Becton Dickinson). Cells which migrated through the reconstituted basement membrane after 24 hours were fixed in methanol, stained with hematoxylin, and counted.

\section{Results}

Integrin binding to extracellular matrix increases association of the intracellular domain with FAK and paxillin $[3,7]$. To determine if FAK and paxillin could interact with the $\beta 1$ integrin subunit in human SCC lines, we immunoprecipitated this receptor from SCC4 and SCC25 cells cultured in suspension, plated on tissue culture plastic, or attached to type IV collagen. As shown in Fig. 1A and 1B, 4 fold more FAK protein associated with $\beta 1$ integrin subunit when cells were plated on both plastic and type IV collagen compared to anchorage deprived cells ( $p<$ $0.00001)$. Preincubation with a blocking antibody to $\beta 1$ integrin but not $\beta 4$ integrin inhibited the association of FAK with $\beta 1$ integrin when cells were plated on type IV collagen. Paxillin was also recruited to the $\beta 1$ integrin complex when cells were plated on plastic ( 2 fold, p < 0.03 ) or type IV collagen ( 5 fold, $\mathrm{p}<0.00001$ ) but not when the cells were anchorage deprived. Incubation with blocking antibody to $\beta 1$ integrin also inhibited the association of paxillin with the integrin subunit in cells plated on type IV collagen ( 5 fold, $\mathrm{p}<0.03$ ). Blocking antibodies failed to inhibit FAK or paxillin recruitment when cells were plated on plastic, indicating that the antibody effectively blocked attachment to type IV collagen. However the blocking antibodies did not inhibit cell attachment since the cells could also adhere to the tissue culture plastic dishes via charge interactions. In contrast, the $52 \mathrm{kd}$ Shc isoform was detected in the $\beta 1$ integrin complex only when cells were anchorage deprived. Shc association was not detected in plated cells regardless of substratum or antibody preincubation $(\mathrm{p}<0.05)$. Grb2 associated with $\beta 1$ integrin under all conditions, indicating that the observed changes in FAK, paxillin, and Shc binding were specific. Similar results were obtained using the SCC4 cell line. These results indicated that FAK and paxillin were recruited to $\beta 1$ integrin when cells were attached to substratum while Shc was associated with the receptor in anchorage deprived cells.

FAK deficient cells are less migratory than their normal counterparts [6], and our data indicated that attachment to type IV collagen increased paxillin association with $\beta 1$ integrin when compared to plastic. To determine if FAK and paxillin association with $\beta 1$ integrin correlated with increased migration, we plated SCC4 and SCC25 cells on plastic or type IV collagen with or without preincubation with integrin blocking antibodies. As shown in Fig. 2, attachment to type IV collagen produced a greater than 2 fold increase in the number of cells which migrated into a blank area of the culture dish. This migration was markedly inhibited by preincubation with blocking antibody to $\beta 1$ integrin but not to $\beta 4$ integrin. Integrin blocking antibodies had no effect on cellular migration on plastic, indicating that the antibodies effectively blocked attachment to extracellular matrix. Similar results were obtained 


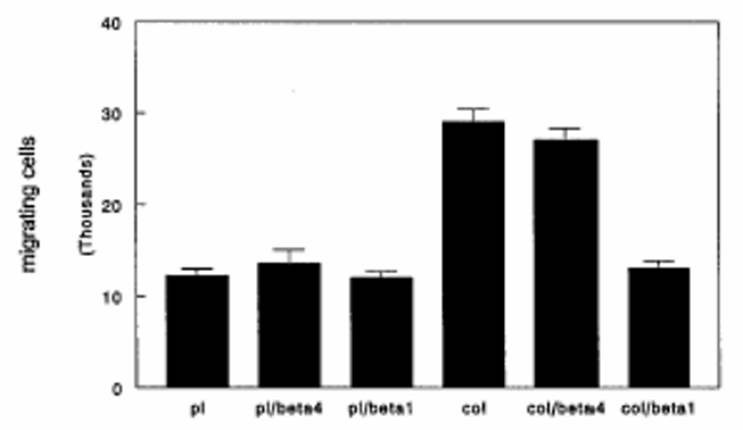

Figure 2

Type IV collagen stimulates $\beta$ I integrin dependent migration of SCC25 cells. SCC25 cells were plated on plastic (pl) tissue culture dishes or type IV collagen (col) coated plates. Some cultures were preincubated with antibodies which block attachment by $\beta$ I or $\beta 4$ integrin subunits. The number of cells which migrated into the blank area of the plate within 24 hours was counted. These experiments were performed three times with similar results. Error bars indicate SEM.

with SCC4 cells. These results indicated that FAK and paxillin recruitment to $\beta 1$ integrin correlated with increased migration on type IV collagen.

FAK overexpression has been associated with increased tumor invasion and lymph node metastasis in clinical cases of human cancer [16]. To determine if FAK and paxillin overexpression produced increased in vitro migration of cancer cell lines, we stably transfected each expression vector into SCC4 and SCC25 cells. FAK and paxillin proteins were up to 20 fold overexpressed in these stable clones (Fig. 3A,3B; p < 0.05). Both FAK and paxillin overexpression effectively induced migration in SCC25 cells (Fig. 3C). FAK overexpression induced migration by 6 fold while paxillin overexpression increased motility by 3 fold when cells were plated on plastic dishes. Migration was further enhanced when cells were plated on type IV collagen. Migration was induced 7 fold when FAK overexpressing cells were plated on type IV collagen compared to controls. Paxillin overexpressing cells were also more migratory on type IV collagen than on plastic; however there was no additive effect of type IV collagen attachment in these clones. Blocking antibody to $\beta 1$ integrin inhibited migration of FAK and paxillin overexpressing clones on type IV collagen but not on plastic. Blocking antibody to $\beta 4$ integrin had no effect on cell migration on either substratum. We were unable to obtain stable clones expressing the $52 \mathrm{kD}$ Shc isoform due to terminal differentiation of these clones (see Discussion). Similar results were obtained with SCC4 cells. These results indicate that FAK and paxillin overexpression promote migration of human
SCC lines which is enhanced by $\beta 1$ integrin attachment to a relevant substratum.

FAK recruitment of paxillin to the cell membrane results in activation of the MAPK pathway [9]. To determine if the MAPK pathway contributed to FAK and paxillin induced migration, we plated these clones on type IV collagen coated dishes and treated the cells with PD98059, a selective upstream inhibitor of ERK1 activation. PD98059 treatment reduced ERK1 activation by up to 3 fold as determined by western blot using an anti-phosphoERK1 antibody (Fig. 4A,4B; p < 0.003). Treatment of FAK and paxillin overexpressing clones with PD98059 inhibited migration on type IV collagen by $75 \%$ (Fig. 4C). However treatment with this inhibitor had little effect on basal migration of control clones, which correlated with our published work [13]. Four to six fold more FAK and paxillin overexpressing cells were able to invade through reconstituted basement membrane in the Matrigel invasion assays (Fig. 4D) which correlated with the migration experiments. MEK inhibition with PD98059 also inhibited invasion by FAK and paxillin overexpressing clones. These results indicated that MAPK inhibition could decrease FAK and paxillin induced migration and invasion by human SCC cells.

\section{Discussion}

The results of this study demonstrated that FAK and paxillin were recruited to the $\beta 1$ integrin subunit when cells were plated on plastic or type IV collagen. However, the $52 \mathrm{kD}$ isoform of the Shc adaptor protein was present in the complex only when cells were maintained under anchorage deprived conditions. Stratified squamous epithelial cells ultimately undergo terminal differentiation when deprived of attachment [17], and $52 \mathrm{kD}$ Shc overexpression was sufficient in our study to produce terminal differentiation of cells plated on plastic tissue culture dishes. These results suggest that localization of $52 \mathrm{kD} \mathrm{Shc}$ to integrin signaling complexes may regulate terminal differentiation of stratified squamous epithelial cells following loss of basement membrane attachment. Shc is known to regulate growth factor receptor induction of the MAPK pathway [18]; it will be interesting to determine if MAPK inhibition prevents anchorage deprived terminal differentiation of stratified squamous epithelial cells.

In this context, FAK has been shown to suppress anchorage deprived cell death in MDCK cells [19]. The kinase activity and autophosphorylation site of the protein is required for this effect. FAK inhibition by overexpression of the carboxyl terminal domain causes loss of adhesion and cell death in human breast cancer lines [20]. Similarly, anchorage independent breast cancer cells undergo apoptosis when FAK function is disrupted. Interestingly, the FAK carboxyl terminus has no effect on adhesion or 


\section{A}

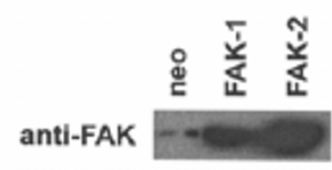

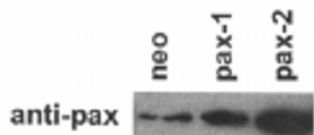

B

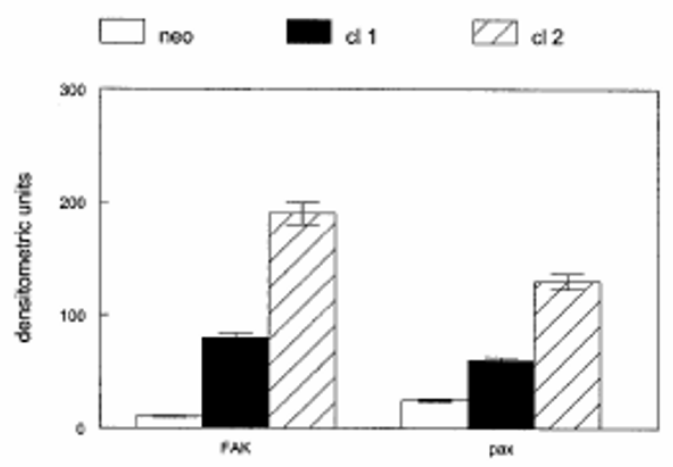

C

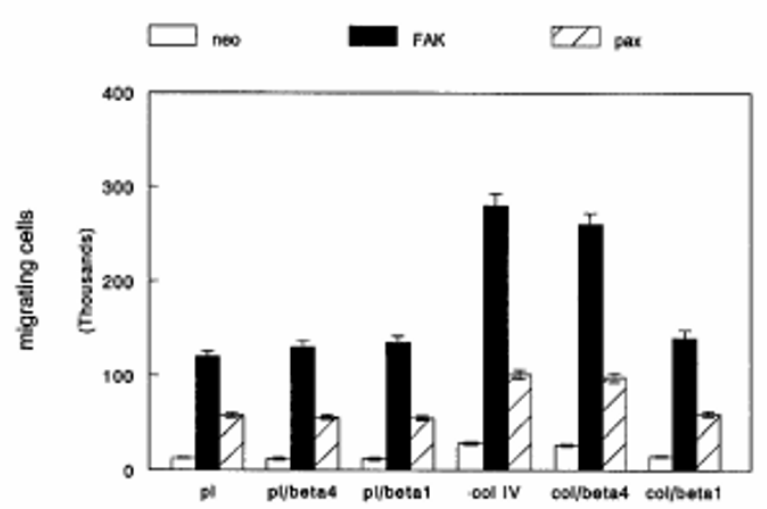

Figure 3

FAK and paxillin promote SCC migration on type IV collagen in a $\beta I$ integrin dependent manner. (A) Overexpression of FAK and paxillin in SCC25 cells. FAK and paxillin expression vectors were stably transfected into SCC25 cells as described in Materials and Methods. FAK and paxillin expression was determined in neomycin resistant control (neo), FAK overexpressing (FAK-I, -2), and paxillin overexpressing (pax-I, -2) clones by western blot using anti-FAK and anti-paxillin (anti-pax) antibodies. This experiment was repeated with additional independently isolated clones with similar results. Representative blots are shown. (B) Densitometric quantitation of blots in Fig. 3A. (C) FAK or paxillin (pax) overexpressing clones or neomycin resistant control cells (neo) were plated on plastic (pl) tissue culture dishes or type IV collagen (col) coated plates. Some cultures were preincubated with antibodies which block attachment by $\beta$ I or $\beta 4$ integrin subunits. The number of cells which migrated into the blank area of the plate within 24 hours was counted. These experiments were performed three times with similar results. Error bars indicate SEM. 


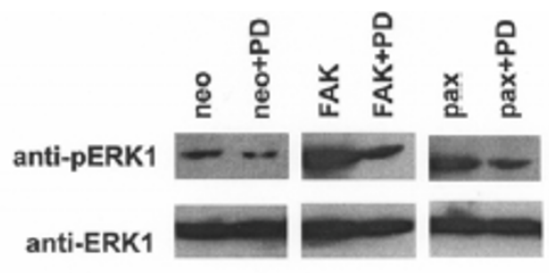

B

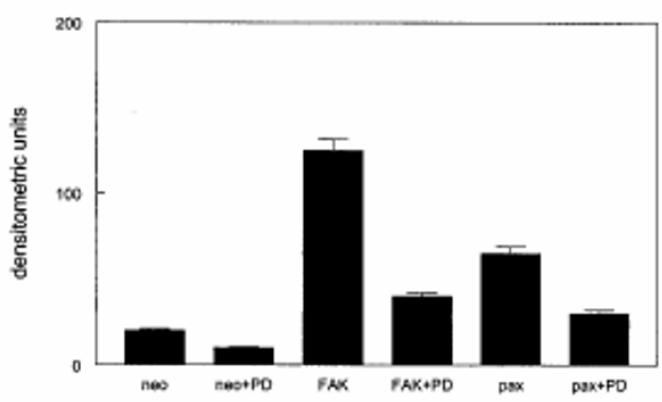

C

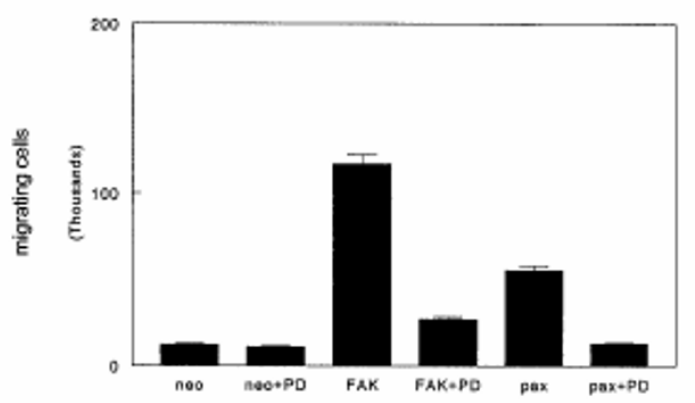

D

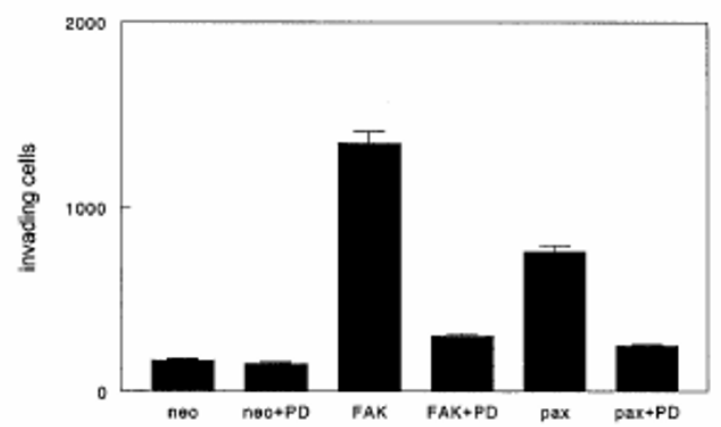

Figure 4

FAK and paxillin induce SCC migration via activation of the mitogen activated protein kinase ERKI. (A) Neomycin resistant control (neo), FAK overexpressing, and paxillin overexpressing (pax) clones were treated with the MEK inhibitor PD98059 (PD) or vehicle as described in Materials and Methods. Relative phosphorylated ERKI and total ERKI expression was determined by western blot using anti-phosphoERKI (anti-pERKI) and anti-ERKI antibodies. This experiment was repeated with additional independently isolated clones with similar results. Representative blots are shown. (B) Densitometric quantitation of blots in Fig. 4A. (C) FAK or paxillin (pax) overexpressing clones or neomycin resistant control cells (neo) were plated on plastic tissue culture dishes and treated with the MEK inhibitor PD98059 (PD) or vehicle as described in Materials and Methods. The number of cells which migrated into the blank area of the plate within 24 hours was counted. (D) FAK or paxillin (pax) overexpressing clones or neomycin resistant control cells (neo) were plated into Matrigel invasion chambers and treated with the MEK inhibitor PD98059 (PD) or vehicle. The number of cells which migrated through the reconstituted basement membrane were fixed, stained, and counted. These experiments were performed three times with similar results. Error bars indicate SEM. 
viability of normal mammary epithelial cells. FAK has been shown to activate JNK which then colocalizes at focal adhesions in primary fibroblasts [21]. This activation occurs via a Ras/Rac1/Pak1/MKK4 pathway. In the presence of serum, survival signals are also transduced by PI3K and Akt. These studies suggest that FAK is also required for survival signaling from the extracellular matrix. Our future studies will examine this property of FAK in human SCC lines.

Our results demonstrated that FAK and paxillin induced migration and invasion of SCC lines was dependent on ERK activity. In colon cancer cell lines, cell attachment to collagen or laminin stimulates phosphorylation of FAK and paxillin and activates ERK1 [22]. FAK inhibition reduces attachment dependent ERK1 activation. ERK1 inhibition reduced migration of colon cancer cells [23], and inhibited growth factor induced migration in SCC lines used in the present study. FAK inhibition also disrupted growth factor stimulated migration of human lung cancer cells [24]. These studies highlight an important role for ERK signaling in mediating collagen induced migration and invasion of human cancer cell lines. FAK expression has been correlated with tumor invasion and lymph node metastasis of cancer cells [16]. In this study, FAK overexpression was detected in 59\% of esophageal cancer pathology specimens. This overexpression was associated with decreased cellular differentiation, late clinical stage, increased depth of invasion, poor survival rate, and lymph node metastasis. Future clinical studies may examine FAK inhibition as potential antitumor therapy.

\section{Conclusions}

FAK and paxillin were recruited to $\beta 1$ integrin when cells were attached to substratum while Shc was associated with the receptor in anchorage deprived cells. FAK and paxillin recruitment to $\beta 1$ integrin correlated with increased migration on type IV collagen. FAK and paxillin overexpression promote migration of human SCC lines which is dependent on $\beta 1$ integrin attachment to its physiologic substratum. MAPK inhibition could decrease FAK and paxillin induced migration and invasion of human SCC cells.

\section{List of abbreviations}

ERK extracellular signal regulated kinase

FAK focal adhesion kinase

Grb2 growth factor receptor bound protein 2

JNK jun N-terminal kinase

PI3K phosphatidylinositol 3-kinase
SCC squamous cell carcinoma

\section{Competing interests}

None declared.

\section{Authors' contributions}

DLC designed the study, supervised cell culture, and drafted the manuscript. AO performed transfections, immunoprecipitation, and western blot experiments. Both authors read and approved the final manuscript.

\section{Acknowledgments}

We thank Dr. Thomas Parsons for expression vectors. This study was supported by National Institutes of Health grant DEI0966.

\section{References}

I. Hynes RO: Cell adhesion: old and new questions. Trends Cell Biol 1999, 9:33-37.

2. Hildebrand JD, Schaller MD, Parsons JT: Paxillin, a tyrosine phosphorylated focal adhesion associated protein binds to the carboxyl terminal domain of focal adhesion kinase. Mol Biol Cell 1995, 6:637-647.

3. Richardson A, Parsons JT: A mechanism for regulation of the adhesion associated protein tyrosine kinase pp I $25 \mathrm{FAK}$. Nature 1996, 380:538-540.

4. Richardson A, Malik RK, Hildebrand JD, Parsons JT: Inhibition of cell spreading by expression of the C-terminal domain of focal adhesion kinase (FAK) is rescued by coexpression of Src or catalytically inactive FAK: a role for paxillin tyrosine phosphorylation. Mol Cell Biol 1997, I7:6906-69|4.

5. Gilmore AP, Romer LH: Inhibition of focal adhesion kinase (FAK) signaling in focal adhesions decreases cell motility and proliferation. Mol Biol Cell I996, 7:I209-I 224.

6. Ilic D, Furuta Y, Kanazawa S, Takeda N, Sobue K, Nakatsuji N, Nomura S, Fukimoto J, Okada M, Yamamoto T, Aizawa S: Reduced cell motility and enhanced focal adhesion contact formation in cells from FAK deficient mice. Nature 1995, 377:539-544.

7. Brown MC, Perrotta JA, Turner CE: Identification of LIM3 as the principal determinant of paxillin focal adhesion localization and characterization of a novel motif on paxillin directing vinculin and focal adhesion kinase binding. J Cell Biol 1996, I35: I I09-I I 23.

8. Thomas JW, Cooley MA, Broome JM, Salgia R, Griffin JD, Lombardo $C R$, Schaller MD: The role of focal adhesion kinase binding in the regulation of tyrosine phosphorylation of paxillin. J Biol Chem 1999, 274:36684-36692.

9. Igishi T, Fukuhara S, Patel V, Katz BZ, Yamada KM, Gutkind JS: Divergent signaling pathways link focal adhesion kinase to mitogen activated protein kinase cascades. J Biol Chem 1999, 274:30738-30746.

10. Hsia DA, Mitra SK, Hauck CR, Streblow DN, Nelson JA, Ilic D, Huang S, Li E, Nemerow GR, Leng J, Spencer KSR, Cheresh DA, Schlaepfer $\mathrm{DD}$ : Differential regulation of cell motility and invasion by FAK. J Cell Biol 2003, I 60:753-767.

II. McLean GW, Brown K, Arbuckle MI, Wyke AW, Pikkarainen T, Ruoslahti E, Frame MC: Decreased focal adhesion kinase suppresses papilloma formation during experimental mouse skin carcinogenesis. Cancer Res 200I, 61:8385-8389.

12. Crowe DL, Tsang KJ, Shemirani B: Jun N-terminal kinase I mediates transcriptional induction of matrix metalloproteinase 9 expression. Neoplasia 2001, 3:27-32.

13. Tsang DK, Crowe DL: The mitogen activated protein kinase pathway is required for proliferation but not invasion of human squamous cell carcinoma lines. Int J Oncol 1999, I 5:519-523.

14. Vo HP, Lee MK, Crowe DL: $\alpha 2 \beta$ I integrin signaling via the mitogen activated protein kinase (MAPK) pathway modulates retinoic acid (RA) dependent downregulation of tumor cell invasion and matrix metalloproteinase 9 activity. Int J Oncol 1998, I3: I I27-I I34. 
15. Wayner EA, Carter WG, Piotrowicz RS, Kunicki T): The function of multiple extracellular matrix receptors in mediating cell adhesion to extracellular matrix. J Cell Biol I988, I07: I88I- I89I.

16. Miyazaki T, Kato H, Nakajima M, Sohda M, Fukai Y, Masuda N, Manda $R$, Fukuchi M, Tsukada K, Kuwano H: FAK overexpression is correlated with tumor invasiveness and lymph node metastasis in esophageal squamous cell carcinoma. Br J Cancer 2003, 89:140-145.

17. Kaur P, Li A: Adhesive properties of human basal epidermal cells: an analysis of keratinocyte stem cells, transit amplifying cells, and postmitotic differentiating cells. J Invest Derm 2000, II 4:413-420.

18. Renshaw MW, Ren XD, Schwartz MA: Growth factor activation of MAP kinase requires cell adhesion. EMBO J 1997, 16:5592-5599.

19. Frisch SM, Vuori K, Ruoslahti E, Chan-Hui PY: Control of adhesiondependent cell survival by focal adhesion kinase. I Cell Biol 1996, 134:793-799.

20. Xu LH, Yang X, Bradham CA, Brenner DA, Baldwin AS Jr, Craven RJ, Cance WG: The focal adhesion kinase suppresses transformation-associated anchorage-independent apoptosis in human breast cancer cells involvement of death receptor-related signalling pathways. J Biol Chem 2000, 275:30597-30604.

21. Almeida EA, Ilic D, Han Q, Hauck CR, Jin F, Kawakatsu H, Schlaepfer $\mathrm{DD}$, Damsky $\mathrm{CH}$ : Matrix survival signaling: from fibronectin via focal adhesion kinase to c-jun $\mathbf{N H}_{(2)}$-terminal kinase. J Cell Biol I49:74I-754.

22. Sanders MA, Basson MD: Collagen IV dependent ERK activation in human Caco-2 intestinal epithelial cells requires focal adhesion kinase. J Biol Chem 2000, 275:38040-38047.

23. Yu CF, Sanders MA, Basson MD: Human Caco-2 motility redistributes FAK and paxillin and activates p38 MAPK in a matrix dependent manner. Am J Physiol Gastrolintest Liver Physiol 2000, 278:G952-66.

24. Hauck CR, Sieg DJ, Hsia DA, Loftus JC, Gaarde WA, Monia BP, Schlaepfer DD: Inhibition of focal adhesion kinase expression or activity disrupts epidermal growth factor stimulated signaling promoting the migration of invasive human carcinoma cells. Cancer Res 200 I, 6 1:7079-7090.

\section{Pre-publication history}

The pre-publication history for this paper can be accessed here:

http://www.biomedcentral.com/1471-2407/4/18/prepub

\section{Publish with Biomed Central and every scientist can read your work free of charge}

"BioMed Central will be the most significant development for disseminating the results of biomedical research in our lifetime. "

Sir Paul Nurse, Cancer Research UK

Your research papers will be:

- available free of charge to the entire biomedical community

- peer reviewed and published immediately upon acceptance

- cited in PubMed and archived on PubMed Central

- yours - you keep the copyright 